Pacific Journal of Mathematic 


\title{
NONCOMMUTATIVE CONVOLUTION MEASURE ALGEBRAS
}

\author{
JOSEPH L. TAYLOR
}

\begin{abstract}
A convolution measure algebra is a partially ordered Banach algebra in which the norm, order, and algebraic operations are related in special ways. Examples include the group algebra $L^{1}(G)$ and measure algebra $M(G)$ on a locally compact group $G$ and, more generally, the measure algebra $M(S)$ on any locally compact semigroup $S$.

This paper demonstrates several ways in which a convolution measure algebra can be realized as an algebra of measures on a compact semigroup. A relationship is established between such realizations and certain classes of Banach space representations of the algebra. These results give a partial extension to the noncommutative case of the structure theory of commutative semi-simple convolution measure algebras.
\end{abstract}

The notion of convolution measure algebra was introduced in [22]. The main theorem of [22] states that a semisimple commutative convolution measure algebra $\mathfrak{M}$ may be represented as an algebra of measures on a compact Abelian topological semigroup $S$, where $S$ has the property that the multiplicative linear functionals on $\mathfrak{M}$ are given by the semicharacters of $S$. In this paper we seek analogous results in the noncommutative case.

The central idea of the paper is this: we attempt to represent a given convolution measure algebra $\mathfrak{M}$ as an algebra of measures on a compact semigroup $S$ in such a way that certain key structural properties of $\mathfrak{M}$ are reflected in the structure of $S$. The map from $\mathfrak{M}$ into the measure algebra $M(S)$ will be called a realization of $\mathfrak{M}$ on $S$. It turns out that for a given convolution measure algebra there are several different semigroup realizations, each of which has intrinsic importance. We motivate the study of semigroup realizations as follows: compact topological semigroups have a very rich structure which has been the object of a great deal of study recently (cf. [1], [12], and [23]); it is hoped that structure theorems for compact semigroups will suggest structure theorems for convolution measure algebras and aid in their proofs. In particular, if $\mathfrak{M}$ has a realization on a semigroup $S$, then the kernel, idempotents, maximal groups, and ideals of $S$ should be related to corresponding structural elements of $\mathfrak{M}$. For a given $\mathfrak{M}$ and $S$ the success of the above program depends on having transportation between $\mathfrak{M}$ and $S$; i.e., one must develop ways of interpreting structure in $S$ in terms of structure in $\mathfrak{M}$ and vice versa. This is where the greatest difficulties in the theory lie. 
For the commutative case the above program shows some promise (cf. [18], [19], [20], [21], and [22]). In this paper we make only a modest beginning on the program for the noncommutative case. We restrict our attention primarily to the problem of constructing semigroup realizations.

In $\S 1$ we collect together the information on $L$-space theory which will be required in the later sections. In $\S 2$ we define the notion of convolution measure algebra and develop a method for constructing realizations. The relationships between Banach space or Hilbert space representations, and realizations for a convolution measure algebra are discussed in $\S 3$. Here we also show the existence of maximal jointly continuous and separately continuous realizations.

1. Preliminaries. The Banach space structure of a convolution measure algebra is that of a complex $L$-space. In this section we recall some of the salient points of the theory of complex $L$-spaces.

Real $L$-spaces were defined by Kakutani in [10] as lattice ordered real Banach spaces in which the norm and order relations satisfy certain conditions. In [15] Rieffel extended the notion to the complex case. For our purpose here, we do not need the abstract definition of $L$-space. It suffices to know that complex $L$-spaces are ordered complex Banach spaces that are characterized by the properties listed below.

1.1. Let $X$ be a locally compact Hausdorff space and $M(X)$ be the ordered Banach space of finite complex regular Borel measures on $X$. An $L$-subspace of $M(X)$ is a closed subspace $\mathfrak{M}$ such that $\mu \in \mathfrak{M}$ and $\nu$ absolutely continuous w.r.t. $\mu$ implies $\nu \in \mathfrak{M}$. Every $L$-subspace of $M(X)$ is a complex $L$-space (cf. [22]).

1.2. If $\mathfrak{M}$ is any complex $L$-space, then there is a compact extremely disconnected Hausdorff space $X$ and an order preserving isometry $\mu \rightarrow \mu_{X}$ of $\mathfrak{M}$ onto a weak-* dense $L$-subspace of $M(X)$ such that every $F \in \mathfrak{M}^{*}$ has the form $F(\mu)=\int f d \mu_{X}$ for some $f \in C(X)$. The correspondence $F \leftrightarrow f$ is an isometry between $\mathfrak{M}^{*}$ and $C(X)$ (cf. [2] and [22]). We shall call $X$ the standard domain of $\mathfrak{M}$.

For a given $L$-space $\mathfrak{M}$, there are many ways of representing $\mathfrak{M}$ in an order-preserving isometric fashion as an $L$-subspace of a measure space $M(Y)$. The above gives a canonical such representation. Certain measure theoretic notions are independent of which of these concrete representations are used; these include real and imaginary part, total variation, absolute continuity, and $L$-subspace. These notions are invariant because they can all be defined purely in terms of the order, linear space structure, and norm on $\mathfrak{M}$. 
1.3. According to 1.2 , the dual $\mathfrak{M}^{*}$ of an $L$-space $\mathfrak{M}$ has in a natural way the structure of a commutative $C^{*}$-algebra with identity (since it may be identified with $C(X)$ ). This $C^{*}$-algebra structure is characterized by the condition that $(\bar{f} \cdot f)(\mu) \geqq 0$ for $0 \leqq \mu \in \mathfrak{M}$ and $f \in \mathfrak{M}^{*}$, where $f \rightarrow \bar{f}$ is the involution (complex conjugation in $C(X)$ ).

Henceforth, we shall always consider the dual of an $L$-space to be an object in the category of commutative $C^{*}$-algebras with identity. A morphism in this category will be a ${ }^{*}$-homomorphism which preserves the identity.

1.4. If $\mathfrak{M}$ and $\mathfrak{N}$ are $L$-spaces and $\varphi: \mathfrak{M} \rightarrow \mathfrak{N}$ is a bounded linear map, then $\varphi$ is a morphism of $L$-spaces (or $L$-homomorphism) if:

(1) $\varphi \mu \geqq 0$ whenever $\mu \geqq 0$;

(2) $\|\varphi \mu\|=\|\mu\|$ if $\mu \geqq 0$, and

(3) $0 \leqq \mu \in \mathfrak{M}$ and $0 \leqq \omega \leqq \varphi \mu$ implies there exists $\nu \in \mathfrak{N}$ such that $\varphi \nu=\omega$ (cf. [22], Definition 1.3).

For a bounded linear map $\varphi: \mathfrak{M} \rightarrow \mathfrak{N}$ the following are equivalent: (a) $\varphi$ is an $L$-homomorphism; (b) $\varphi^{*}: \mathfrak{R}^{*} \rightarrow \mathfrak{M}^{*}$ is a ${ }^{*}$-homomorphism preserving identities; (c) $\varphi^{* *}: \mathfrak{M}^{* *} \rightarrow \mathfrak{R}^{* *}$ is an $L$-homomorphism, (cf. [22], Th. 1.2). Hence, with this definition of $L$-space morphism $\mathfrak{M} \rightarrow \mathfrak{M}^{*}$ is a contravariant functor from the category of $L$-spaces to the category of commutative $C^{*}$-algebras with identity.

It turns out that if an $L$-homomorphism $\varphi: \mathfrak{M} \rightarrow \mathfrak{N}$ is injective, then it is an order preserving isometry of $\mathfrak{M}$ onto an $L$-subspace of $\Re$ (cf. [22]).

Since the dual $\varphi^{*}: \mathfrak{R}^{*} \rightarrow \mathfrak{M}^{*}$ of an $L$-homomorphism $\varphi: \mathfrak{M} \rightarrow \mathfrak{R}$ is a *-homomorphism preserving the identity, it follows that $\phi^{*}$ induces a continuous map $\alpha: X \rightarrow Y$, where $X$ and $Y$ are the standard domains of $\mathfrak{M}$ and $\mathfrak{N}$ respectively. The maps $\varphi, \varphi^{*}$, and $\alpha$ are related by

$$
\int_{X} \varphi^{*} f d \mu=\varphi^{*} f(\mu)=f(\varphi \mu)=\int_{Y} f d \phi \mu
$$

and $\varphi^{*} f(x)=f(\alpha(x))$ for $f \in \mathfrak{N}^{*}=C(Y), \mu \in \mathfrak{M} \subset M(X)$, and $x \in X$. Here, we have taken the liberty-as we shall often do-of identifying certain spaces with their images under canonical isomorphisms. This greatly simplifies notation with no great loss of clarity (we hope).

1.5. If $\mathfrak{M}$ is an $L$-space, then the $C^{*}$-algebra structure of $\mathfrak{M}^{*}$ can be most readily identified in applications in the following way: if $0 \leqq \mu \in \mathfrak{M}$, then there is a natural order preserving isometry $\varphi_{\mu}=$ $(f \rightarrow f d \mu)$ of $L^{1}(\mu)$ into $\mathfrak{M}$. The dual $\varphi_{\mu}^{*}$ of this map is a map of $\mathfrak{M}^{*}$ onto $L^{1}(\mu)^{*}=L^{\infty}(\mu)$. That $\phi_{\mu}^{*}$ be a $C^{*}$-algebra homomorphism for each $\iota$ is a condition characterizing the $C^{*}$-algebra structure of $\mathfrak{M}^{*}$. 
1.6. If $\mathfrak{M}$ and $\mathfrak{R}$ are $L$-spaces, then there is a pair consisting of an $L$-space $\mathfrak{M} \otimes \mathfrak{R}$ and a bilinear $\operatorname{map}(\mu, \nu) \rightarrow \mu \otimes \nu: \mathfrak{M} \times \mathfrak{N} \rightarrow \mathfrak{M} \otimes \mathfrak{N}$ such that the following condition holds: $\left({ }^{*}\right)$ if $X$ and $Y$ are locally compact Hausdorff spaces and $\mu \rightarrow \mu_{X}, \nu \rightarrow \nu_{Y}$ order preserving isometries of $\mathfrak{M}$ and $\mathfrak{N}$ onto $L$-subspaces of $M(X)$ and $M(Y)$ respectively, then $\mu \otimes \nu \rightarrow \mu_{X} \times \nu_{Y}$ determines an order preserving isometry of $\mathfrak{M} \otimes \mathfrak{N}$ onto the closed linear span in $M(X \times Y)$ of the measures $\mu_{X} \times \nu_{Y}(\mu \in \mathfrak{M}, \nu \in \mathfrak{R})$. The space $\mathfrak{M} \otimes \mathfrak{R}$ can be defined as the closed linear span in $M(X \times Y)$ of the measures $\mu \times \nu(\mu \in \mathfrak{M}, \nu \in \mathfrak{N})$, where $X$ and $Y$ are the standard domains of $\mathfrak{M}$ and $\mathfrak{N}$ respectively (cf. [22]). It is then quite easy to show that $\left(^{*}\right)$ holds even if $X$ and $Y$ are not the standard domains.

2. Convolution measure algebras and semigroup realizations. We defined the class of convolution measure algebras in [22]. For convenience, we restate the definition in an equivalent form below:

Definition 2.1. A convolution measure algebra is a complex $L$ space $\mathfrak{M}$ together with an $L$-homomorphism $\varphi: \mathfrak{M} \otimes \mathfrak{M} \rightarrow \mathfrak{M}$ which is associative $(\varphi(\mu \otimes \varphi(\nu \otimes \omega))=\varphi(\varphi(\mu \otimes \nu) \otimes \omega))$.

Under the operation $(\mu, \nu) \rightarrow \mu \cdot \nu=\varphi(\mu \otimes \nu)$, a convolution measure algebra is a Banach algebra. We shall use the abbreviation C.M.A. for the term convolution measure algebra. If $\mathfrak{M}$ is a C.M.A. then an $L$-subalgebra of $\mathfrak{M}$ is a subalgebra which is also an $L$-subspace (§1.1). An $L$-subalgebra of a C.M.A. is again a C.M.A.

The interesting examples of C.M.A.'s arise as $L$-subalgebras of the measure algebra $M(S)$ on a topological group or semigroup. By a topological semigroup $S$ we shall mean a semigroup with a Hausdorff topology such that for each $t \in S$ the maps $s \rightarrow s t$ and $s \rightarrow t s$ are continuous from $S$ to $S$ (separate continuity). If $(s, t) \rightarrow s t: S \times S \rightarrow S$ is also continuous, then $S$ will be called a jointly continuous topological semigroup.

In [22] we proved that if $S$ is a locally compact jointly continuous topological semigroup, then $M(S)$ is a C.M.A. under convolution multiplication. The same thing is true even if the multiplication in $S$ is not jointly continuous. However, the proof is more subtle due to the possible nonmeasurability of a separately continuous function on a product space. The next two lemmas are designed to circumvent this difficulty.

Let $X$ and $Y$ be locally compact Hausdorff spaces. We denote by $C^{\omega}(X \times Y)$ the space of bounded separately continuous functions $f$ on $X \times Y$ (i.e., $x \rightarrow f\left(x, y_{0}\right)$ and $y \rightarrow f\left(x_{0}, y\right)$ are continuous for each fixed $x_{0} \in X, y_{0} \in Y$ ). Using Grothendieck's weak compactness theorem (cf. 
[7], Th. 5), Glicksberg in [6] proves that if $f \in C^{\omega}(X \times Y), \mu \in M(X)$, $v \in M(Y)$, then

$$
\int f(x, y) d \mu(x) \text { and } \int f(x, y) d \nu(y)
$$

are continuous functions of $y$ and $x$ respectively and

$$
\iint f(x, y) d \mu(x) d \nu(y)=\iint f(x, y) d \nu(y) d \mu(x) .
$$

Note that this may not imply the existence of the double integral $\int f d \mu \times \nu$. We require a sharpened form of this theorem.

Lemma 2.1. Let $X$ and $Y$ be locally compact Hausdorff spaces, $f \in C^{\omega}(X \times Y), 0 \leqq \mu \in M(X)$, and $0 \leqq \nu \in M(Y)$. Then there exists a unique element $g \in L^{\infty}(\mu \times \nu)$ such that

$$
\begin{aligned}
0 & =\iint|f(x, y)-g(x, y)| d \mu(x) d \nu(y) \\
& =\iint|f(x, y)-g(x, y)| d \nu(y) d \mu(x) ;
\end{aligned}
$$

i.e., $f$ and $g$ agree almost everywhere on $x$ or $y$ cross-sections.

Proof. Without loss of generality we may assume $X$ and $Y$ are compact. If $f \in C^{\omega}(X \times Y)$ then function $\left|f(x, y)-f\left(x^{\prime}, y\right)\right|$ is bounded, continuous in $\left(x, x^{\prime}\right) \in X \times X$ for each $y \in Y$, and continuous in $y \in Y$ for each $\left(x, x^{\prime}\right) \in X \times X$. It follows from Glicksberg's result that the function

$$
h\left(x, x^{\prime}\right)=\int\left|f(x, y)-f\left(x^{\prime}, y\right)\right| d \nu(y)
$$

is continuous on $X \times X$. Since $h(x, x)=0$ for every $x$ and the diagonal in $X \times X$ is compact, there exist for each $k$ points $x_{1}, \cdots, x_{n} \in X$ and an open cover $\left\{U_{1}, \cdots, U_{n}\right\}$ of $X$ such that $x_{i} \in U_{i}$ and $h\left(x, x^{\prime}\right)<k^{-1}$ for $\left(x, x^{\prime}\right) \in U_{i} \times U_{i}$.

Let $\varphi_{1}, \cdots, \varphi_{n}$ be a partition of unity in $\mathrm{C}(X)$ subordinate to the open cover $\left\{U_{1}, \cdots, U_{n}\right\}$. We set $g_{k}(x, y)=\sum \varphi_{i}(x) f\left(x_{i}, y\right)$. Note that $g_{k} \in C(X \times Y)$ and

$$
\begin{aligned}
& \iint\left|f(x, y)-g_{k}(x, y)\right| d \mu(x) d \nu(y) \\
& \quad=\iint\left|f(x, y)-g_{k}(x, y)\right| d \nu(y) d \mu(x) \\
& \quad \leqq \sum \int \varphi_{i}(x) \int\left|f(x, y)-f\left(x_{i} y\right)\right| d \nu(y) d \mu(x)
\end{aligned}
$$




$$
=\sum \int \varphi_{i}(x) h\left(x, x_{i}\right) d \mu(x) \leqq \frac{\|\mu\|}{k}
$$

It follows that $\left\{g_{k}\right\}$ coverges in $L^{1}(\mu \times \nu)$-norm to an element $g \in L^{\infty}(\mu \times \nu)$ which has the properties we require. The uniqueness is trivial.

Note that the lemma does not directly imply that $f$ is $\mu \times \nu$ measurable. Whether or not this must be the case seems to be an open question. However, the lemma makes this question almost irrelevant for most purposes.

The correspondence $f \rightarrow g$ of Lemma 2.1 is clearly a $C^{*}$-algebra homomorphism of $C^{\omega}(X \times Y)$ into $L^{\infty}(\mu \times \nu)$ which extends the natural homomorphism of $C(X \times Y)$ into $L^{\infty}(\mu \times \nu)$. If $\mathfrak{M}$ and $\mathfrak{N}$ are $L$ subspaces of $M(X)$ and $M(Y)$ respectively, then $\mathfrak{M} \otimes \mathfrak{N}$ can be identified with the linear span in $M(X \times Y)$ of the product measures $\mu \times \nu(\mu \in \mathfrak{M}, \nu \in \mathfrak{R})$ (cf. $\S 1.6)$. It follows from this and $\S 1.5$ that there is a natural $C^{*}$-algebra homomorphism $\eta: C^{\omega}(X \times Y) \rightarrow(\mathfrak{M} \otimes \Re)^{*}$ which satisfies

$$
\eta f(\mu \otimes \nu)=\iint f(x, y) d \mu(x) d \nu(y)
$$

For $\eta$ to be injective it is obviously necessary and sufficient that $\mathfrak{M}$ and $\mathfrak{N}$ be weak-* dense in $M(X)$ and $M(Y)$ respectively. Hence, we have proved:

LEMMA 2.2. If $X$ and $Y$ are locally compact Hausdorff spaces and $\mathfrak{M}$ and $\mathfrak{R}$ are weak-* dense $L$-subspaces of $M(X)$ and $M(Y)$ respectively, then there is $a^{*}$-isomorphism $\eta$ of $C^{\omega}(X \times Y)$ onto a subalgebra of $(\mathfrak{M} \otimes \mathfrak{N})^{*}$ such that

$$
\eta f(\mu \otimes \nu)=\iint f(x, y) d \mu(x) d \nu(y)=\iint f(x, y) d \nu(y) d \mu(x) .
$$

THEOREM 2.1. If $S$ is a locally compact topological semigroup, then the equation

$$
\int h d(\mu \cdot \nu)=\iint h(s t) d \mu(s) d \nu(t) \quad\left(f \in C_{0}(S)\right)
$$

defines a multiplication on $M(S)$ under which it is a convolution measure algebra.

Proof. Glicksberg proved in [6] that equation (2.1) defines a multiplication on $M(S)$ which makes it a Banach algebra. To show that $M(S)$ is a convolution measure algebra, we will show that this multiplication is defined by an $L$-homomorphism $\varphi: M(S) \otimes M(S) \rightarrow M(S)$.

Let $C_{b}(S)$ denote the algebra of bounded continuous functions on $S$. 
We define a *-algebra homomorphism $\theta: C_{b}(S) \rightarrow(M(S) \otimes M(S))^{*}$ as the map $h \rightarrow f: C_{b}(S) \rightarrow C^{\omega}(S \times S)$ (where $f(s, t)=h(s \cdot t)$ ) followed by the map $\eta: C^{\omega}(S \times S) \rightarrow(M(S) \otimes M(S))^{*}$ of Lemma 2.2. Notice that equation (2.1.) simply says that

$$
\int h d(\mu \cdot \nu)=\theta h(\mu \otimes \nu) \text {. }
$$

If $\varphi$ is the dual $\theta^{*}:(M(S) \otimes M(S))^{* *} \rightarrow C_{b}(S)^{*}$ of $\theta$ restricted to $M(S)$ $\otimes M(S)$, then $\varphi$ is an $L$-homomorphism (cf. $\S 1.4$ ), since $\theta$ is a *-homomorphism preserving identities. Since $\mu \cdot \nu=\varphi(\mu \otimes \nu), M(S)$ satisfies Definition 2.1 and is a convolution measure algebra.

The morphisms in the category of convolution measure algebras are defined in part (a) of the following definition.

Definition 2.2. (a ) A map $\theta: \mathfrak{M} \rightarrow \mathfrak{N}$ between C.M.A.'s $\mathfrak{M}$ and $\mathfrak{R}$ will be called a C.M.A.-homomorphism if it is both an algebraic homomorphism and an $L$-homomorphism between $\mathfrak{M}$ and $\mathfrak{R}$ as $L$-spaces.

(b) A realization of $\mathfrak{M}$ on a locally compact topological semigroup $S$ is a C.M.A.-homomorphism $\theta: \mathfrak{M} \rightarrow M(S)$ with weak-* dense range. If $\theta$ is injective it will be called a strict realization. If $S$ is compact, $\theta$ will be called a compact realization.

The purpose of the paper is to demonstrate the existence of certain compact realizations of a convolution measure algebra $\mathfrak{M}$. In practice, a C.M.A. $\mathfrak{M}$ will usually be given as an $L$-subalgebra of the measure algebra on a group or semigroup. In this situation we already have one semigroup realization of $\mathfrak{M}$ in hand. However, the given semigroup may not reflect strongly the structure of $\mathfrak{M}$. Hence, it is worthwhile to seek another realization in which the underlying semigroup has a richer structure. In the commutative case, this is illustrated by the algebra $M(G)$, where $G$ is a nondiscrete 1.c.a. group. It is not true that every complex homomorphism of $M(G)$ is determined by a character of $G$; however, there is a realization of $M(G)$ on a compact jointly continuous semigroup $S$ such that every complex homomorphism of $M(G)$ is determined by a semi-character of $S$ (cf. [22]). We seek similar "structurally rich" realizations in the noncommutative case.

Throughout the remainder of this section $\mathfrak{M}$ will denote a C.M.A. with multiplication map $\varphi: \mathfrak{M} \otimes \mathfrak{M} \rightarrow \mathfrak{M}$ and standard domain $X$ (cf. $\S 1.2$ ). We shall consider $\mathfrak{M}$ to be an $L$-subspace of $M(X)$ and $\mathfrak{M} \otimes \mathfrak{M}$ to be the closed linear span in $M(X \otimes X)$ of the product measures $\mu \times \nu(\mu, \nu \in \mathfrak{M})$.

According to Lemma 2.2, we may consider the algebra $C^{\omega}(X \otimes X)$ of separately continuous bounded functions to be a subalgebra of 
$(\mathfrak{M} \otimes \mathfrak{M})^{*}$. Thus, we shall simultaneously consider an element of $C^{\omega}(X \times X)$ (or $C(X \times X) \subset C^{\omega}(X \times X)$ ) as a function on $X \times X$ and as a linear functional on $\mathfrak{M} \otimes \mathfrak{M}$.

If $f \in C(X)=\mathfrak{M}^{*}$ then $\varphi^{*} f \in(\mathfrak{M} \otimes \mathfrak{M})^{*}$ may or may not lie in the subalgebra $C^{\omega}(X \times X)$. If it does lie in $C^{\omega}(X \times X)$ then it makes sense to talk about its cross sections $\left(\varphi^{*} f\right)_{x}$ and $\left(\varphi^{*} f\right)^{x}$, where $\left(\varphi^{*} f\right)_{x}(y)=\varphi^{*} f(x, y)$ and $\left(\varphi^{*} f\right)^{x}(y)=\varphi^{*} f(y, x)$. For each $x \in X$ these cross sections will be elements of $C(X)$.

THEOREM 2.2. Let $A$ be a closed *-subalgebra of $\mathfrak{M}^{*}=C(X)$ containing the identity. There is a compact realization $\theta: \mathfrak{M} \rightarrow M(S)$ of $\mathfrak{M}$ such that $\theta^{*}: M(S)^{*} \rightarrow \mathfrak{M}^{*}$ maps $C(S)$ isomorphically onto $A$ if and only if $A$ satisfies the following conditions:

(1) $\varphi^{*}$ maps $A$ into $C^{\omega}(X \times X)$;

(2) if $f \in A$ then $\left(\varphi^{*} f\right)_{x} \in A$ and $\left(\varphi^{*} f\right)^{x} \in A$ for every $x \in X$.

The semigroup $S$ has a jointly continuous multiplication if and only if $\varphi^{*} A \subset C(X \times X)$. The realization $\theta$ is strict if and only if $A$ is weak-* dense in $\mathfrak{M}^{*}$.

Proof. With minor differences the proof is the same as the proof of Theorem 2.2 of [22]. Hence, we settle for a brief outline here.

If $S$ is the maximal ideal space of the commutative $C^{*}$-algebra $A$, then $S$ is a compact Hausdorff space and $A \approx C(S)$. Conditions (1) and (2) on $A$ insure that $\varphi^{*}: A \rightarrow C^{\omega}(X \times X)$ may be considered a map from $C(S)$ into $C^{\omega}(S \times S)$. It follows that $\varphi^{*}$ induces a separately continuous semigroup operation on $S$. The realization $\theta: \mathfrak{M} \rightarrow M(S)$ is defined in the obvious way noting that $M(S)=A^{*}$ and $A \subset \mathfrak{M}^{*}$.

DeFinition 2.3. For a given realization $\theta: \mathfrak{M} \rightarrow M(S)$, the algebra $A=\theta^{*} C(S) \subset \mathfrak{M}^{*}$ of Theorem 2.2 will be denoted $A_{\theta}$.

Obviously, the algebra $A_{\theta}$ completely determines the realization $\theta$. The following theorem has a trivial proof which we omit; however, it is probably worth stating explicitly:

THEOREM 2.3. Let $\theta_{1}: \mathfrak{M} \rightarrow M\left(S_{1}\right)$ and $\theta_{2}: \mathfrak{M} \rightarrow M\left(S_{2}\right)$ be compact realizations of $\mathfrak{M}$. The relation $A_{\theta_{1}} \subset A_{\theta_{2}}$ holds if and only if there is a continuous surjective homomorphism $\alpha: S_{2} \rightarrow S_{1}$ such that the diagram

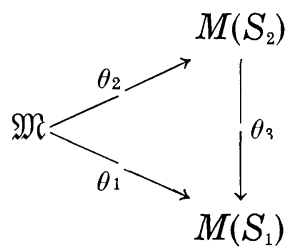


is commutative, where $\theta_{3} \mu(E)=\mu\left(\alpha^{-1}(E)\right)$.

An example of an interesting semigroup realization is the realization constructed in [22] for a commutative C.M.A. M. Here the algebra $A_{f}$ is the closed linear span in $\mathfrak{M}^{*}$ of the complex homomorphisms of $\mathfrak{M}$.

3. Representations. In this section we will demonstrate the existence of semigroup realizations in which the structure of the underlying semigroup $S$ is sufficiently rich to allow a certain class of Banach space representations of $\mathfrak{M}$ to be viewed as arising from representations of $S$.

We are motivated by the work of Eberlein in [3], De Leeuw and Glicksberg in [4], and Loomis in [11], on almost periodic functions.

Throughout this section $\mathfrak{M}$ will denote a convolution measure algebra with standard domain $X$. If $\mathfrak{U}$ is a Banach space, then $B(\mathfrak{H})$ will denote the algebra of bounded linear operators on $\mathfrak{A}$. A left representation of $\mathfrak{M}$ on $\mathfrak{U}$ is a bounded homomorphism $\mu \rightarrow T_{\mu}$ of $\mathfrak{M}$ into $B(\mathfrak{U})$. A right representation of $\mathfrak{M}$ on $\mathfrak{U}$ is a bounded antihomomorphism $\mu \rightarrow T_{\mu}$ of $\mathfrak{M}$ into $B(\mathfrak{U})$. Most of our results will be stated for left representations only, though analogous results hold for right representations. We shall use the term "representation" to mean "left representation" unless otherwise specified. For a comprehensive study of representation theory for Banach algebras see [14].

There are natural left and right representations $\mu \rightarrow L_{\mu}$ and $\mu \rightarrow R_{\mu}$ of $\mathfrak{M}$ on $\mathfrak{M}^{*}$ defined by $L_{\mu \prime} f(\nu)=f(\nu \mu)$ and $R_{\mu} f(\nu)=f(\mu \nu)$ for $f \in \mathfrak{M}^{*}$ and $\mu, \nu \in \mathfrak{M}$. If $\mu \rightarrow T_{\mu}$ is any representation of $\mathfrak{M}$ on a Banach space $\mathfrak{A}$, then a subspace $\mathfrak{A}_{1}$ of $\mathfrak{A}$ is said to be invariant under $\mu \rightarrow T_{t}$ if $T_{\mu} \mathfrak{A}_{1} \subset \mathfrak{A}_{1}$ for every $\mu \in \mathfrak{M}$. A subspace $A$ of $\mathfrak{M}^{*}$ is said to be left (righ) invariant if it is invariant under $\mu \rightarrow L_{\mu}\left(\mu \rightarrow R_{\mu}\right)$. If we set $N(\mathfrak{I})=\left\{f \in \mathfrak{M}^{*}: f(\mu)=0\right.$ for every $\left.\mu \in \mathfrak{\Im}\right\}$, then $\mathfrak{\Im} \leftrightarrow N(\mathfrak{I})$ defines a one-to-one correspondence between the set of all closed right (left) ideals $\mathfrak{\Im}$ of $\mathfrak{M}$ and the set of all weak-* closed left (right) invariant subspaces of $\mathfrak{M}^{*}$.

Definition 3.1. If $\mu \rightarrow T_{\mu}$ is a representation of $\mathfrak{M}$ on $\mathfrak{A}$, then an element $\xi \in \mathfrak{A}$ will be called almost periodic (under $\mu \rightarrow T_{\mu}$ ) if $\left\{T_{\mu} \xi: \mu \in \mathfrak{M},\|\mu\| \leqq 1\right\}$ has compact closure in the norm of $\mathfrak{A}$. If $\left\{T_{\mu} \xi: \mu \in \mathfrak{M},\|\mu\| \leqq 1\right\}$ has compact closure in the weak topology of $\mathfrak{A}$, then $\xi$ will be called weakly almost periodic. If every $\xi \in \mathfrak{A}$ is almost periodic (weakly almost periodic), we will say that $\mu \rightarrow T_{\mu}$ is an almost periodic (weakly almost periodic) representation. An element $f \in \mathfrak{M}^{*}$ will be called left almost periodic (left weakly almost periodic) if it is almost periodic (weakly almost periodic) under $\mu \rightarrow L_{\mu}$ and 
right almost periodic (right weakly almost periodic) if it is almost periodic (weakly almost periodic) under $\mu \rightarrow R_{\mu}$.

The above definitions are adaptations of definitions used in [4].

LEMMA 3.1. If $\mu \rightarrow T_{\mu}$ is a weakly almost periodic representation of $\mathfrak{M}$ on $\mathfrak{X}$, then there is a map $x \rightarrow T_{x}$ of $X$ into $B(\mathfrak{U})$ such that $x \rightarrow T_{x}$ is continuous from $X$ to $B(\mathfrak{X})$ with the weak operator topology and $\eta\left(T_{\mu} \xi\right)=\int_{X} \eta\left(T_{x} \xi\right) d \mu(x)$ for $\mu \in \mathfrak{M}, \xi \in \mathfrak{N}$, and $\eta \in \mathfrak{U} *$ If $\mu \rightarrow T_{\mu}$ is almost periodic, then $x \rightarrow T_{x}$ is continuous from $X$ to $B(\mathfrak{U})$ with the strong operator topology.

Proof. For fixed $\xi \in \mathfrak{A}$ denote the map $\mu \rightarrow T_{\mu} \xi$ by $\psi$. Then $\psi^{* *}$ is a map from $\mathfrak{M}^{* *}=M(X)$ into $\mathfrak{U}^{* *}$ which is continuous from the weak-* topology on $M(X)$ to the weak-* topology on $\mathfrak{A}^{* *}$. The weak-* closure of the unit ball of $\mathfrak{M}$ in $M(X)$ is the unit ball of $M(X)$ and so $\psi^{* *}$ maps this onto the weak-* closure in $\mathfrak{U}^{* *}$ of

$$
\{\psi \mu: \mu \in \mathfrak{M},\|\mu\| \leqq 1\}=\left\{T_{\mu} \xi: \mu \in \mathfrak{M},\|\mu\| \leqq 1\right\} .
$$

However, since $\mu \rightarrow T_{\mu}$ is weakly almost periodic $\left\{T_{u} \xi: \mu \in \mathfrak{M},\|\mu\| \leqq 1\right\}$ has weakly compact closure in $\mathfrak{A}$ and so its closure in $\mathfrak{A}$ agrees with its weak-* closure in $\mathfrak{U}^{* *}$. Thus, the range of $\psi^{* *}$ lies in $\mathfrak{A}$. If $\lambda \in M(X)$, we denote $\psi^{* *} \lambda$ by $U_{\lambda} \xi$. Clearly $U_{\lambda} \xi$ depends linearly on $\xi$, and $\lambda \rightarrow U_{\lambda}$ is a bounded linear map of $M(X)$ into $B(\mathfrak{U})$ which is continuous from the weak-* topology of $M(X)$ to the weak operator topology of $B(\mathfrak{U})$. If we set $T_{x}=U_{\hat{o}_{x}}$ where $\delta_{x}$ is the point mass at $x$, then the continuity of $\lambda \rightarrow U_{\lambda}$ implies $\eta\left(U_{\lambda} \xi\right)=\int_{X} \eta\left(T_{x} \xi\right) d \lambda(x)$ for $\lambda \in M(X), \xi \in \mathfrak{Y}$, and $\eta \in \mathfrak{Y}^{*}$. Since $T_{\mu}=U_{\mu}$ for $\mu \in \mathfrak{M}$, the map $x \rightarrow T_{x}$ has the required properties.

If $\mu \rightarrow T_{\mu}$ is almost periodic, then the map $\psi^{* *}$ of the above argument carries the unit ball of $M(X)$ onto a norm compact subset of $\mathfrak{A}$. On this set the weak and norm topologies agree. Hence $\psi^{* *}$ is continuous, on the unit ball of $M(X)$, from the weak-* topology of $M(X)$ to the norm topology of $\mathfrak{T}$. It follows that $\curvearrowright \rightarrow T_{x}$ is continuous from $X$ to $B(\mathfrak{U})$ with the strong operator topology.

In the following lemma $\varphi: \mathfrak{M} \otimes \mathfrak{M} \rightarrow \mathfrak{M}$ denotes the multiplication map of $\mathfrak{M}$, as in $\S 2$.

Lemma 3.2. If $f \in \mathfrak{M}^{*}=C(X)$ then the following statements are equivalent:

(1) $\varphi^{*} f \in C(X \times X)\left(C^{\omega}(X \times X)\right)$;

(2) $f$ is left almost periodic (left weakly almost periodic);

(3) $f$ is right almost periodic (right weakly almost periodic); 
(4) there is an almost periodic (weakly almost periodic) representation $\mu \rightarrow T_{\mu}$ of $\mathfrak{M}$ on a Banach space $\mathfrak{A}$ and $\left\{\xi \in \mathfrak{A}, \eta \in \mathfrak{A}^{*}\right\}$ such that $f(\mu)=\eta\left(T_{\mu} \xi\right)$.

Proof. We shall show that $(1) \Rightarrow(2) \Rightarrow(4) \Rightarrow(1)$. Since statement (1) is symmetric it follows that $(1) \Leftrightarrow(3)$ is also true.

To show that (1) implies (2) we note first that if $\varphi^{*} f \in C^{\omega}(X \times X)$ and $\mu \in \mathfrak{M}$, then $L_{\mu} f(x)=\int_{X} \varphi^{*} f(x, y) d \mu(y)$, since

$$
L_{\mu} f(\nu)=f(\nu \mu)=\int_{X} \int_{X} \varphi^{*} f(x, y) d \mu(y) d \nu(x)
$$

for $\nu \in \mathfrak{M}$. Also, if $\varphi^{*} f \in C^{\omega}(X \times X)$, then the map $y \rightarrow\left(\phi^{*} f\right)_{y}$, where $\left(\varphi^{*} f\right)_{y}(x)=\varphi^{*} f(x, y)$, is continuous from $X$ to $C(X)$ with the topology of pointwise convergence on $X$. By Grothendieck's weak compactness theorem (cf. [7], Th. 5), $y \rightarrow\left(\varphi^{*} f\right)_{y}$ is also continuous from $X$ to $C(X)$ with the weak topology. It follows that if we define $U_{\lambda} f(x)=$ $\int_{X} \int_{X} \varphi^{*} f(x, y) d \lambda(y)$ for $\lambda \in M(X)$, then $\lambda \rightarrow U_{\lambda} f$ is continuous from $M(X)$ with the weak-* topology to $X$ with the weak topology. Since the unit ball of $M(X)$ is weak-* compact and $L_{\mu}=U_{\mu}$ for $\mu \in \mathfrak{M}$, we have that $\left\{L_{\mu} f: \mu \in \mathfrak{M},\|\mu\| \leqq 1\right\}$ has weakly compact closure in $C(X)$. Hence $f$ is left weakly almost periodic. If $\phi^{*} f \in C(X \times X)$, then the map $y \rightarrow\left(\phi^{*} f\right)_{y}$ is continuous from $X$ to $C(X)$ with the norm topology, the map $\lambda \rightarrow U_{y}$ is continuous from $M(X)$ with the weak-* topology to $C(X)$ with the norm topology, $\left\{L_{\mu} f: \mu \in \mathfrak{M},\|\mu\| \leqq 1\right\}$ has norm compact closure, and $f$ is left almost periodic.

To show that (2) implies (4) we first assume that $\mathfrak{M}$ has an identity $\zeta$. If $f$ is left almost periodic (left weakly almost periodic), then the norm closure $\mathfrak{A}$ in $\mathfrak{M}^{*}$ of $\left\{L_{\mu} f: \mu \in \mathfrak{M}\right\}$ is a left invariant subspace of $\mathfrak{M}^{*}$ in which every element is left almost periodic (left weakly almost periodic). Therefore, the representation $\mu \rightarrow L_{\mu}$, restricted to this subspace, is an almost periodic (weakly almost periodic) representation of $\mathfrak{M}$. Furthermore, we have that

$$
f(\mu)=f(\zeta \mu)=\left(L_{\mu} f\right)(\zeta) .
$$

If $\mathfrak{M}$ does not have an identity, we go to the algebra $\mathfrak{M}^{\prime}$ obtained by adjoining an identity $\zeta$ to $\mathfrak{M}$. If $X^{\prime}$ is the standard domain of $\mathfrak{M}^{\prime}$, then $X^{\prime}=X \cup\left\{x_{0}\right\}$, where $x_{0}$ is a discrete point of $X^{\prime}$ and $\zeta$ is the point mass at $x_{0}$. We may identify $\mathfrak{M}^{*}=C(X)$ as the space of functions in $\left(\mathfrak{M}^{\prime}\right)^{*}=C\left(X^{\prime}\right)$ which vanish at $x_{0}$. It follows that $f$ is left almost periodic (left weakly almost periodic) as an element of $\mathfrak{M}^{*}$ if and only if it has the same property as an element of ( $\left.\mathfrak{M}^{\prime}\right)^{*}$. Replacing $\mathfrak{M}$ by $\mathfrak{M}^{\prime}$ in the above paragraph leads to the conclusion that $(2) \Rightarrow(4)$ in general. 
That $(4) \Rightarrow(1)$ follows from Lemma 3.1, since $f(\mu)=\eta\left(T_{\mu} \zeta\right)$ implies that $f(x)=\eta\left(T_{x} \zeta\right)$ and $\phi^{*} f(x, y)=\eta\left(T_{x} T_{y} \zeta\right)$. Since multiplication in the unit ball of $B(\mathfrak{A})$ is separately continuous in the weak operator topology and jointly continuous in the strong operator topology; we have $\varphi^{*} f \in C^{\omega}(X \times X)(C(X \times X))$ if $\mu \rightarrow T_{\mu}$ is almost periodic (weakly almost periodic).

Theorem 2.2 may be restated in terms of the representations $\mu \rightarrow L_{\mu}$ and $\mu \rightarrow R_{\mu}$ as follows:

Theorem 3.1. If $A$ is a subset of $\mathfrak{M}^{*}$, then $A=A_{\theta}$ for some compact realization $\theta$ of $\mathfrak{M}$ if and only if the following conditions hold:

(1) $A$ is a closed subalgebra of $\mathfrak{M}^{*}$ which is closed under conjugation and contains the constant functions,

(2) every $f \in A$ is weakly almost periodic,

(3) $A$ is invariant under both the right representation $\mu \rightarrow R_{\mu}$ and the left representation $\mu \rightarrow L_{\mu}$ of $\mathfrak{M}$ on $\mathfrak{M}^{*}$.

The semigroup $S$ is jointly continuous if and only if every $f \in A$ is almost periodic.

Proof. In view of Theorem 2.2 and Lemma 3.2, we need only show that in the presence of the other conditions, condition (2) of Theorem 2.2 holds if and only if condition (3) of Theorem 3.1 holds. To see this, recall that in the proof of Lemma 3.2 we showed that if $f$ is weakly almost periodic and we set $U_{\lambda} f(x)=\int_{X} \varphi^{*} f(x, y) d \lambda(y)$ for $\lambda \in M(X)$, then $U_{\mu} f=L_{\mu} f$ for $\mu \in \mathfrak{M}$ and the map $\lambda \rightarrow U_{\lambda} f$ is continuous from $M(X)$ with the weak-* topology to $\mathfrak{M}^{*}$ with the weak topology. Thus, if $A$ is a closed subspace, it follows that $L_{\mu} f \in A$ for every $\mu \in \mathfrak{M}$ if and only if $U_{k} f \in A$ for every $\lambda \in M(X)$; and this holds if and only if $\left(\varphi^{*} f\right)^{x} \in A$ for every $x \in X$, where $\left(\varphi^{*} f\right)^{x}(y)=$ $\varphi^{*} f(x, y)$. An analogous argument shows that $R_{\mu} f \in A$ for every $\mu \in \mathfrak{M}$ if and only if $\left(\varphi^{*} f\right)_{x} \in A$ for every $x \in X$. Hence $A$ is both left and right invariant if and only if $f \in A$ implies $\left(\varphi^{*} f\right)^{x} \in A$ and $\left(\varphi^{*} f\right)_{x} \in A$ for $x \in X$.

A standard theorem in the study of group algebras is that every representation of such an algebra on a reflexive Banach space is induced by a representation of the underlying group (cf. [11], §32). We now develop an analogue of this result.

DEFINITION 3.2. A representation of a topological semigroup $S$ on a Banach space $\mathfrak{A}$ is a bounded homomorphism $s \rightarrow T_{s}$ of $S$ into the multiplicative semigroup of $B(\mathfrak{H})$ such that $s \rightarrow T_{s}$ is continuous 
from $S$ to $B(\mathfrak{U})$ with the weak operator topology. If $s \rightarrow T_{s}$ is continuous from $S$ to $B(\mathfrak{U})$ with the strong operator topology, then $s \rightarrow$ $T_{s}$ will be called a strongly continuous representation.

DEFINITION 3.3. If $\mu \rightarrow T_{\mu}$ is a reprsentation of $\mathfrak{M}$ on $\mathfrak{A}$ and $\theta$ is a realization of $\mathfrak{M}$ on $S$, then $\mu \rightarrow T_{\mu}$ will be called $\theta$-induced if there is a representation $s \rightarrow T_{s}$ of $S$ on $\mathfrak{A}$ such that

$$
\eta\left(T_{\mu} \xi\right)=\int_{S} \eta\left(T_{s} \xi\right) d \theta \mu(s) \text { for } \xi \in \mathfrak{A}, \eta \in \mathfrak{A}^{*} .
$$

If $s \rightarrow T_{s}$ is strongly continuous, then $\mu \rightarrow T_{\mu}$ will be called strongly $\theta$-induced.

THEOREM 3.2. If $\mu \rightarrow T_{\mu}$ is a representation of $\mathfrak{M}$ on $\mathfrak{A}$ and $\theta$ is a compact realization of $\mathfrak{M}$ on $S$, then $\mu \rightarrow T_{\mu}$ is $\theta$-induced if and only if $\mu \rightarrow T_{\mu}$ is weakly almost periodic and each element $f \in \mathfrak{M}^{*}$, of the form $f(\mu)=\eta\left(T_{\mu} \xi\right)$ for $\xi \in \mathfrak{Y}$ and $\eta \in \mathfrak{X} *$, is in $A_{\theta}$. Also, if $\mu \rightarrow T_{\mu}$ is $\theta$-induced, then it is strongly $\theta$-induced if and only if $\mu \rightarrow T_{\mu}$ is almost periodic.

Proof. Since $\theta^{*}: C(S) \rightarrow A_{\theta} \subset C(X)$ is a *algebra isomorphism, it is induced by a surjective map $\alpha$ from $X$ to $S$. Then $A_{\theta}$ consists of those elements $f \in \mathfrak{M}^{*}$ of the form $f(x)=g(\alpha(x))$ for $g \in C(S)$, i.e., those elements $f \in \mathfrak{M}^{*}$ for which $\alpha(x)=\alpha(y)$ implies $f(x)=f(y)$. If $\mu \rightarrow T_{n}$ is weakly almost periodic, let $x \rightarrow T_{x}$ be the map from $X$ to $B(\mathfrak{A})$ given by Lemma 3.1. If for each $\xi \in \mathfrak{U}^{*}$ the element $f \in \mathfrak{M}^{*}$, defined by $f(\mu)=\eta\left(T_{\mu} \xi\right)$, is in $A$, then $\alpha(x)=\alpha(y)$ implies $\eta\left(T_{x} \xi\right)=$ $f(x)=f(y)=\eta\left(T_{y} \xi\right)$. It follows that $\alpha(x)=\alpha(y)$ implies $T_{x}=T_{y}$, and $x \rightarrow T_{x}$ defines a map $s \rightarrow T_{s}$ of $S$ into $B(\mathfrak{U})$ such that $T_{x}=T_{\alpha(x)}$. Then

$$
\eta\left(T_{\mu} \xi\right)=\int_{X} \eta\left(T_{x} \xi\right) d \mu(x)=\int_{S} \eta\left(T_{s} \xi\right) d \theta \mu(s)
$$

for $\mu \in \mathfrak{M}, \xi \in \mathfrak{A}, \eta \in \mathfrak{A}^{*}$. The map $s \rightarrow T_{s}$ is continuous into $B(\mathfrak{U})$ with the weak operator topology since $x \rightarrow T_{x}$ is continuous. That $s \rightarrow T_{s}$ is a homomorphism follows from the equations

$$
\begin{aligned}
& \int_{S} \int_{S} \eta\left(T_{s} T_{t} \xi\right) d \theta \mu(s) d \theta \nu(t)=\eta\left(T_{\mu} T_{\nu} \xi\right) \\
& \quad=\eta\left(T_{\mu . \nu} \xi\right)=\int_{S} \int_{S} \eta\left(T_{s t} \xi\right) d \theta \mu(s) d \theta \nu(t)
\end{aligned}
$$

for $\mu, \nu \in \mathfrak{M}, \xi \in \mathfrak{A}, \eta \in \mathfrak{U}^{*}$.

If $\mu \rightarrow T_{\mu}$ is almost periodic, then $x \rightarrow T_{x}$ and thus $s \rightarrow T_{s}$ are continuous maps into $B(\mathfrak{A})$ with the strong operator topology.

Conversely, if $\mu \rightarrow T_{\mu}$ is $\theta$-induced, then there is a representation $s \rightarrow T_{s}$ of $S$ on $\mathfrak{A}$ such that 


$$
\eta\left(T_{\mu} \xi\right)=\int_{S} \eta\left(T_{s} \xi\right) d \theta \mu(s) \text { for } \quad \mu \in \mathfrak{M}, \xi \in \mathfrak{U}, \eta \in \mathfrak{X}^{*}
$$

Since $S$ is compact, it follows that $\mu \rightarrow T_{\mu}$ is weakly almost periodic, and if $s \rightarrow T_{s}$ is strongly continuous, then $\mu \rightarrow T_{\mu}$ is almost periodic. If $f(\mu)=\eta\left(T_{\mu} \xi\right)$, then $f(x)=g(\alpha(x))$ where $g$ is the function in $C(S)$ defined by $g(s)=\eta\left(T_{s} \xi\right)$. Thus $f \in A_{\theta}$ and the proof is complete.

We can now state our main theorem.

THEOREM 3.3. There are compact semigroup realizations $\omega: \mathfrak{M} \rightarrow$ $M\left(S_{\omega}\right)$ and $\sigma: \mathfrak{M} \rightarrow M\left(S_{o}\right)$ such that the following conditions hold:

(1) $A_{w}$ is the space of all weakly almost periodic functions in $\mathfrak{M}^{*}$ and $A_{\sigma}$ is the space of all almost periodic functions in $\mathfrak{M}^{*}$;

(2) Every weakly almost periodic representation of $\mathfrak{M}$ is $\omega$ induced and every almost periodic representation of $\mathfrak{M}$ is strongly $\sigma$-induced;

(3) $S_{\sigma}$ is jointly continuous.

Proof. It follows from Lemma 3.2 that the spaces of almost periodic and weakly almost periodic functions are *-subalgebras of $\mathfrak{M}^{*}$ containing the identity. Both subalgebras are clearly invariant under each $L_{\mu}$ and each $R_{\mu}$. Hence, the theorem follows directly from Theorems 3.1 and 3.2 .

COROLLARY. Every representation of $\mathfrak{M}$ in a reflexive Banach space is $\omega$-induced. Every representation of $\mathfrak{M}$ in a finite dimensional Banach space is $\sigma$-induced.

Corollary. A C.M.A. M has a strict compact realization if and only if the weakly almost periodic elements of $\mathfrak{M}^{*}$ are weak-* dense. It has a strict compact realization on a jointly continuous semigroup if and only if the almost periodic elements of $\mathfrak{M}^{*}$ are weak-* dense.

If there are sufficiently many representations of $\mathfrak{M}$ in reflexive (finite dimensional) Banach spaces to separate points of $\mathfrak{M}$, then $\mathfrak{M}$ has a strict compact (jointly continuous) realization.

Note that Theorems 2.3 and 3.2 imply the realizations $\omega: \mathfrak{M} \rightarrow$ $M\left(S_{\omega}\right)$ and $\sigma: \mathfrak{M} \rightarrow M\left(S_{\sigma}\right)$ are maximal compact and compact jointly continuous realizations in the obvious sense. That is any compact realization of $\mathfrak{M}$ can be factored through $\omega$ and any compact jointly continuous realization can be factored through $\sigma$.

We now turn briefly to the study of *-representations of a convolution measure algebra $\mathfrak{M}$ with involution. It is natural in this 
situation to insist that the involution respect the $L$-space structure of $\mathfrak{M}$.

DEFINITION 3.3. A convolution measure algebra with involution is a convolution measure algebra $\mathfrak{M}$ with an algebra involution $\mu \rightarrow \mu^{*}$ which satisfies the following additional condition: if $\mu^{\prime}=\bar{\mu}^{*}$ (the complex conjugate of $\left.\mu^{*}\right)$, then $\mu \rightarrow \mu^{\prime}$ is an $L$-homomorphism of $\mathfrak{M}$ onto $\mathfrak{M}$.

If $G$ is a locally compact group, then $M(G)$ is a convolution measure algebra with involution, where $\mu^{*}(E)=\overline{\mu\left(E^{-1}\right)}$. Here the $L$-homomorphism $\mu \rightarrow \mu^{\prime}$ is defined by $\mu^{\prime}(E)=\mu\left(E^{-1}\right)$. More generally, let $S$ be a locally compact topological semigroup with an involution $s \rightarrow s^{\prime}$, i.e., a map $s \rightarrow s^{\prime}$ which is a continuous anti-isomorphism of $S$ with $s^{\prime \prime}=s$. If we set $\mu^{\prime}(E)=\mu\left(E^{\prime}\right)$ and $\mu^{*}=\overline{\mu^{\prime}}$, then $M(S)$ becomes a convolution measure algebra with involution.

Throughout the remainder of this section, $\mathfrak{M}$ will denote a fixed convolution measure algebra with involution. $A *$-realization of $\mathfrak{M}$ will be a realization $\theta$ on a semigroup $S$ with involution $s \rightarrow s^{\prime}$ such that $\theta \mu^{*}(E)=\overline{\theta \mu\left(E^{\prime}\right)}$. We shall obtain a result relating *-realizations to *-representations of $\mathfrak{M}$ on a Hilbert space $H$.

Lemma 3.3. There are natural involutions on $S_{\omega}$ and $S_{\sigma}$ such that $\omega$ and $\sigma$ are ${ }^{*}$-realizations.

Proof. The map $\mu \rightarrow \mu^{\prime}=\overline{\mu^{*}}$ is an $L$-homomorphism and, hence, it induces an algebra isomorphism $f \rightarrow f^{\prime}$ on $C(X)$ such that $f\left(\mu^{\prime}\right)=$ $f^{\prime}(\mu)$. The lemma will follow if we can show that $A_{\omega}$ and $A_{\sigma}$ are invariant under this isomorphism. The required involution $s \rightarrow s^{\prime}$ on $S_{\omega}\left(\operatorname{resp} . S_{\sigma}\right)$ is then induced in the obvious manner by $f \rightarrow f^{\prime}$ on $A_{\omega} \approx C\left(S_{\omega}\right)$ (resp. $A_{\sigma} \approx C\left(S_{\sigma}\right)$ ).

Let $x \rightarrow x^{\prime}$ denote the homeomorphism of $X$ such that $f^{\prime}(x)=f\left(x^{\prime}\right)$. If $f \in A_{\omega}$ then by Lemma 3.2, $\varphi^{*} f \in C^{\omega}(X \times X)$. A simple computation shows that the function $g(x, y)=\phi^{*} f\left(y^{\prime}, x^{\prime}\right)$ on $X \times X$ determines the linear functional $\varphi^{*} f^{\prime}$ on $\mathfrak{M} \otimes \mathfrak{M}$. It now follows from Lemma 3.2 that $f \in A_{\omega}$ implies $f^{\prime} \in A_{\omega}$ and $f \in A_{\sigma}$ implies $f^{\prime} \in A_{\sigma}$. This completes the proof.

Definition 3.5. Let $A_{\rho}$ be the closed linear span in $\mathfrak{M}^{*}=C(X)$ of linear functionals of the form $f(\mu)=\left(A_{\mu} h, h\right)$, where $\mu \rightarrow A_{\mu}$ is a *-representation of $\mathfrak{M}$ on a Hilbert space $H$ and $h \in H$. Let $A$, be the closed linear span in $\mathfrak{M}^{*}$ of the functionals of the form $f(\mu)=$ $\left(A_{\mu} h, h\right)$ such that $\mu \rightarrow A_{\mu}$ is an irreducible *-representation of $\mathfrak{M}$ on $H$ and $h \in H$. 
The functionals described above are the states and pure states respectively of the *-algebra $\mathfrak{M}$. Note that $A_{\iota} \subset A_{\rho} \subset A_{\omega}$, since a Hilbert space is reflexive.

If $S$ is a semigroup with involution $s \rightarrow s^{\prime}$, then a *-representation of $S$ on a Hilbert space $H$ is a weakly continuous homomorphism $s \rightarrow U_{s}$ of $S$ into the multiplicative semigroup of $B(H)$ such that $U_{s^{\prime}}=$ $U_{s}^{*}$. If $\mu \rightarrow A_{\mu}$ is a ${ }^{*}$-representation of $\mathfrak{M}$, then by the first corollary to Theorem 3.3 it is induced by a representation $s \rightarrow U_{s}$ of $S_{\omega}$. It is clear that $s \rightarrow U_{s}$ is a ${ }^{*}$-representation of $S_{\omega}$. Hence, *-representation of $\mathfrak{M}$ are all induced by *-representation of $S_{\omega}$. Similarly, irreducible *-representations of $\mathfrak{M}$ are induced by irreducible *-representations of $S_{\omega}$. However, there may not be enough *-representations (or irreducible *-representations) of $S_{\omega}$ to separate points. One can obtain the following result by factoring out equivalence relations in $S_{\omega}$ :

THEOREM 3.4. There are compact ${ }^{*}$-realizations $\rho$ and $\iota$ of $\mathfrak{M}$ on semigroups $S_{\rho}$ and $S_{c}$ respectively, such that:

(1) $A_{\rho}=\rho^{*} C\left(S_{o}\right)$ and $A_{i}=\iota^{*} C\left(S_{i}\right)$;

(2) the *-representation of $\mathfrak{M}$ are all $\rho$-induced by *-representations of $S_{\rho}$ and the irreducible *-representations of $\mathfrak{M}$ are all $\iota$ induced by irreducible *-representations of $S_{i}$;

(3) the *-representations of $S_{\rho}$ separate points in $S_{\rho}$ and the

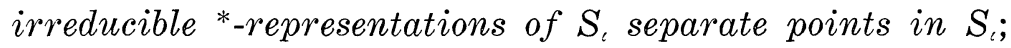

(4) $\rho$ and chave the same kernel.

Part (4) of the above theorem follows from the fact that the weak-* closed convex hull of the set of normalized pure states is the set of all normalized states (cf. [14]).

An interesting problem in connection with Theorem 3.4 is the following: when is it true that the involution on $S_{\rho}$ or $S_{c}$ is normal in the sense that $s^{\prime} s=s s^{\prime}$ for all $s$ ? If a semigroup $S$ has a normal involution and $s \rightarrow U_{s}$ is a *-representation of $S$, then each $U_{s}$ is a normal operator, $U_{p}$ is a projection if $p \in S$ is an idempotent, an $U_{s}$ is a partial isometry if $s$ lies in a subgroup of $S$. Obviously then, the study of *-representations of a semigroup $S$ will be considerably simpler if $S$ has a normal involution.

4. Conclusions and problems. We do not have satisfactory conditions on an abstract convolution measure algebra $\mathfrak{M}$ which guarantee that $\mathfrak{M}$ has a strict realization on a compact semigroup. This seems to be a hard problem. However, from our point of view it is largely irrelevant. The theory initiated here is intended for use as a tool in the study of the representation theory of specific convolution measure algebras such as $M(G)$. As is the case with $M(G)$, one may 
expect to have a-priori knowledge that the algebra he is studying has sufficiently many representations in a reflexive Banach space to separate points. Of course, the algebra then has a strict realization on $S_{\omega}$ by the second corollary to Theorem 3.3.

Given the work we have done here, one should now be able to use the structure theory of compact semigroups and semigroup representations both as a tool in proving results about convolution measure algebras (such as $M(G)$ ) and as an intuition aid in deciding what these results should be. The monograph of Berglund and Hofmann [1] and the fundamental paper of Glicksberg and DeLeeuw [4] should be useful in this connection. We shall leave this project to someone else.

If $\theta: \mathfrak{M} \rightarrow M(S)$ is a compact semigroup realization then there are some trivial results concerning the connection between structure in $\mathfrak{M}$ and structure in $S$ that can be carried over directly from results in [22] for the commutative case. We will close by mentioning some of these without proof.

If $\mathfrak{M}$ has an approximate identity $\left\{\mu_{\alpha}\right\}$ with $\left\|\mu_{\alpha}\right\| \leqq 1$ (or $\mu_{\alpha} \geqq 0$ ) for each $\alpha$, then $S$ has an identity (cf. [22], Theorem 3.1).

If every one-dimensional representation of $\mathfrak{M}$ is $\theta$-induced, then there is a one-to-one correspondence between prime $L$-ideals of $\mathfrak{M}$ and open-compact prime ideals of $S_{\theta}$ (cf. [22], Th. 3.2).

If $\mathfrak{M}=L^{1}(G)$ for a locally compact group $G$, then $S_{\omega}$ is the weakly almost periodic compactification of $G$ and $S_{\sigma}$ is the almost periodic compactification of $G$ (cf. [22], $\S 4)$. If $\mathfrak{M}=M(G)$ then $S_{\omega}$ and $S_{\sigma}$ contain as closed ideals copies of the corresponding semigroups for $L^{1}(G)$ (cf. [22], §4).

\section{REFERENCES}

1. J. F. Berglund and K. H. Hofmann, Compact semitopological semigroups and weakly almost periodic functions, Springer-Verlag, 1967.

2. F. Cunningham, Jr., L-structure in L-spaces, Trans. Amer. Math. Soc. 95 (1960), 274-299.

3. W. F. Eberlein, Abstract ergodic theorems and weakly almost periodic functions, Trans. Amer. Math. Soc. 67 (1949), 217-240.

4. K. de Leeuw and I. Gliksberg, Applications of almost periodic compactifications, Acta Math. 105 (1961), 63-97.

5. - Almost periodic functions on semigroups, Acta Math. 105 (1961) 99-140.

6. I. Glicksberg, Weak compactness and separate continuity, Pacific J. Math. 11 (1961), 205-214.

7. A. Grothendieck, Criteres de compacite' daus les espaces fonctionels généraux, Amer.

J. Math. 74 (1952), 168-186.

8. E. Hewitt and S. Kakutani, Some multiplicative linear functionals on $M(G)$, Ann. of Math. 79 (1964), 489-505.

9. E. Hewitt and K. A. Ross, Abstract harmonic analysis, Vol. 1, Grundlehren der Math. Wiss., Band 115, Springer-Verlog, Heidelberg, 1963.

10. S. Kakutani, Concrete representations of abstract L-spaces and the mean ergodic 
theorem, Ann. of Math. (2) 42 (1941), 523-537.

11. L. H. Loomis, An introduction to abstract harmonic analysis, Van Nostrand, New York, 1953.

12. P. S. Mostert, The structure of topological semigroups-revisited, Bull. Amer. Math. Soc. 72 (1966), 601-618.

13. M. A. Naimark, Normed rings, P. Noordhoff N. V., Groningen, The Netherlands, 1959.

14. C. E. Rickart, General theory of Banach algebras, Van Nostrand, Princeton, 1960.

Van Nostrand, Princeton, 1960.

15. M. A. Rieffel, A characterization of commutative group algebras and measure algebras, Trans. Amer. Math. Soc. 119 (1965), 150-166.

16. W. Rudin, Fourier analysis on groups, Interscience, New York, 1962.

17. Yu. A. Sreider, The structure of maximal ideals in rings of measures uith convolution, Amer. Math. Transl. (1) 8 (1962), 365-391.

18. J. L. Taylor, Convolution measure algebras with group maximal ideal spaces, Trans. Amer. Math. Soc. 128 (1967), 257-263.

19. - Ideal theory and Laplace transforms for a class of measure algebras on a group, Acta Math. 121 (1968). 251-292.

20. - L-subalgebras of $M(G)$, Trans. Amer. Math. Soc. 135 (1969), 105-113.

21. The Shilov boundary of the algebra of measures on a group, Proc. Amer. Math. Soc. 16 (1965), 941-945.

22. The structure of convolution measure algebras, Trans. Amer. Math. Soc. 119 (1965), 150-166.

23. A. D. Wallace, The structure of topological semigroups, Bull. Amer. Math. Soc. 61 (1955), 95-112.

Received October 25, 1966, and in revised form June 2, 1969. Research partially sponsored by the Air Force Office of Scientific Research, Office of Aerospace Research, United States Air Force, under AFOSR grant No. 1313-67.

UNIVERSITY OF UTAH 


\title{
PACIFIC JOURNAL OF MATHEMATICS
}

\author{
EDITORS
}

\author{
H. ROYDEN \\ Stanford University \\ Stanford, California \\ RICHARD PIERCE \\ University of Washington \\ Seattle, Washington 98105
}

\author{
J. DUGUNDJI \\ Department of Mathematics \\ University of Southern California \\ Los Angeles, California 90007 \\ BASIL GORDON \\ University of California \\ Los Angeles, California 90024
}

\section{ASSOCIATE EDITORS}

E. F. BECKENBACH
B. H. NeumanN

F. WOLF
K. YoshidA

\section{SUPPORTING INSTITUTIONS}

\author{
UNIVERSITY OF BRITISH COLUMBIA \\ CALIFORNIA INSTITUTE OF TECHNOLOGY \\ UNIVERSITY OF CALIFORNIA \\ MONTANA STATE UNIVERSITY \\ UNIVERSITY OF NEVADA \\ NEW MEXICO STATE UNIVERSITY \\ OREGON STATE UNIVERSITY \\ UNIVERSITY OF OREGON \\ OSAKA UNIVERSITY \\ UNIVERSITY OF SOUTHERN CALIFORNIA
}

\author{
STANFORD UNIVERSITY \\ UNIVERSITY OF TOKYO \\ UNIVERSITY OF UTAH \\ WASHINGTON STATE UNIVERSITY \\ UNIVERSITY OF WASHINGTON

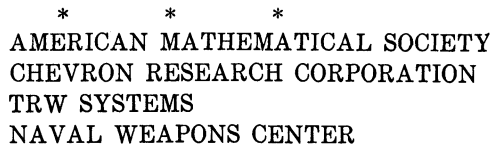

The Supporting Institutions listed above contribute to the cost of publication of this Journal, but they are not owners or publishers and have no responsibility for its content or policies.

Mathematical papers intended for publication in the Pacific Journal of Mathematics should be in typed form or offset-reproduced, double spaced with large margins. Underline Greek letters in red, German in green, and script in blue. The first paragraph or two must be capable of being used separately as a synopsis of the entire paper. It should not contain references to the bibliography. Manuscripts, in duplicate if possible, may be sent to any one of the four editors. Please classify according to the scheme of Math. Rev. 36, 1539-1546. All other communications to the editors should be addressed to the managing editor, Richard Arens, University of California, Los Angeles, California, 90024.

50 reprints are provided free for each article; additional copies may be obtained at cost in multiples of 50 .

The Pacific Journal of Mathematics is published monthly. Effective with Volume 16 the price per volume (3 numbers) is $\$ 8.00$; single issues, $\$ 3.00$. Special price for current issues to individual faculty members of supporting institutions and to individual members of the American Mathematical Society: $\$ 4.00$ per volume; single issues $\$ 1.50$. Back numbers are available.

Subscriptions, orders for back numbers, and changes of address should be sent to Pacific Journal of Mathematics, 103 Highland Boulevard, Berkeley, California, 94708.

PUBLISHED BY PACIFIC JOURNAL OF MATHEMATICS, A NON-PROFIT CORPORATION

Printed at Kokusai Bunken Insatsusha (International Academic Printing Co., Ltd.), 7-17, Fujimi 2-chome, Chiyoda-ku, Tokyo, Japan. 


\section{Pacific Journal of Mathematics}

\section{Vol. 31, No. $3 \quad$ BadMonth, 1969}

George E. Andrews, On a calculus of partition functions .................. 555

Silvio Aurora, A representation theorem for certain connected rings ............ 563

Lawrence Wasson Baggett, A note on groups with finite dual spaces ............. 569

Steven Barry Bank, On majorants for solutions of algebraic differential equations in regions of the complex plane ............................... 573

Klaus R. Bichteler, Locally compact topologies on a group and the corresponding continuous irreducible representations ......................... 583

Mario Borelli, Affine complements of divisors ....................... 595

Carlos Jorge Do Rego Borges, A study of absolute extensor spaces ............. 609

Bruce Langworthy Chalmers, Subspace kernels and minimum problems in Hilbert

spaces with kernel function ...................................... 619

John Dauns, Representation of L-groups and F-rings................. 629

Spencer Ernest Dickson and Kent Ralph Fuller, Algebras for which every

indecomposable right module is invariant in its injective envelope ...........

Robert Fraser and Sam Bernard Nadler, Jr., Sequences of contractive maps and fixed

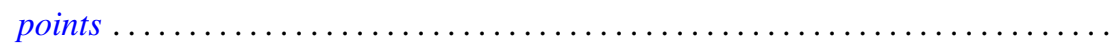

Judith Lee Gersting, A rate of growth criterion for universality of regressive

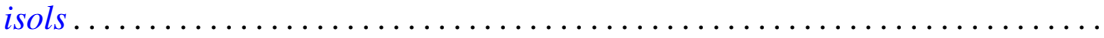

Robert Fred Gordon, Rings in which minimal left ideals are projective ............

Fred Gross, Entire functions of several variables with algebraic derivatives at certain algebraic points

W. Charles (Wilbur) Holland Jr. and Stephen H. McCleary, Wreath products of ordered permutation groups .........................

W. J. Kim, The Schwarzian derivative and multivalence .................. 717

Robert Hamor La Grange, Jr., On $(\mathrm{m}-\mathrm{n})$ products of Boolean algebras ......... 725

Charles D. Masiello, The average of a gauge ........................ 733

Stephen H. McCleary, The closed prime subgroups of certain ordered permutation

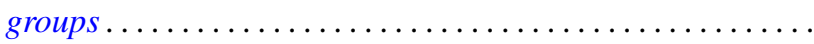

Richard Roy Miller, Gleason parts and Choquet boundary points in convolution measure algebras ...............................

Harold L. Peterson, Jr., On dyadic subspaces ........................ 773

Derek J. S. Robinson, Groups which are minimal with respect to normality being intransitive........................................... 777

Ralph Edwin Showalter, Partial differential equations of Sobolev-Galpern type . . . 787

David Slepian, The content of some extreme simplexes ................... 795

Joseph L. Taylor, Noncommutative convolution measure algebras ............. 809

B. S. Yadav, Contractions of functions and their Fourier series ............... 827

Lindsay Nathan Childs and Frank Rimi DeMeyer, Correction to automorphisms of separable algebras" ....................... 833

Moses Glasner and Richard Emanuel Katz, Correction to: "Function-theoretic degeneracy criteria for Riemannian manifolds".............

Satish Shirali, Correction to: "On the Jordan structure of complex Banach

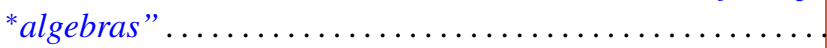

